\title{
Financial difficulties and solutions for industrial transfer under the integration of Beijing-Tianjin-Hebei region
}

\author{
Dong Jianping \\ Department of Finance, Tianjin Agricultural University, Tianjin 300384, China \\ e-mail address: dongjianping68@163.com. *corresponding author: Dong Jianping
}

Keywords: Beijing-Tianjin-Hebei region; Integration; Industrial transfer; Financial difficulties

\begin{abstract}
The integration of Beijing-Tianjin-Hebei region has become a national strategy, and the industrial transfer is imperative. It requires coordinated development of these three regions. However, the big difference of financial service level among these regions makes the industrial transfer slow down. In the present study, the financial difficulties of Beijing-Tianjin-Hebei regional integration are analyzed. The results show that the main obstacles about the regional economic integration are the fuzzy orientation of finance function, the unbalanced financial level and the lack of unified industry standard. Three solutions of broadening the financial channels, speeding up the financial innovation and improving the financial environment are put forward based on the above analyses.
\end{abstract}

\section{Introduction}

As the core of the Bohai economic zone, the Beijing-Tianjin-Hebei region has some congenital advantages of connected region due to similar culture and the complementary resource ${ }^{[1]}$. With the establishment of the third economic growth pole, a large number of domestic and foreign funds, talents and technologies flock to Beijing-Tianjin-Hebei region. Under the guidance of national policy and the pushing of marketing forces, the regional economic development has been accelerated. It is necessary to realize the integrated coordinated development of the Beijing-Tianjin -Hebei region ${ }^{[2]}$. However, the regional barriers have formed due to the long term administrative division. Currently, there is no really economic interdependent relationship among Beijing, Tianjin and Hebei region. Large population continuously flocked to Beijing, the capital of China, resulting in a serious city disease, such as traffic jams, environmental pollution and haze weather ${ }^{[3]}$. Although as early as 10 years ago the government of Beijing, Tianjin and Hebei published a "Langfang consensus" relating to the regional integration ${ }^{[4]}$, the thought barriers of the "small patch of land just your own", the administrative barriers of "my site that I make decision", and the interests fetter of "rich water should be kept in one's own fields", leading to different understandings for the governments of the three regions to the regional integration ${ }^{[5]}$. Since Xi Jinping personally promoted the coordinated development of Beijing- Tianjin-Hebei before two sessions in 2014, the coordinated development for the three regions has become a national strategy. The integrative development of the Beijing-Tianjin-Hebei region has received high attention again, the industrial transfer and undertaking has been carrying out quietly ${ }^{[6]}$.

Industrial transfer refers to a process of certain industries transfer from one country or region to another country or region ${ }^{[7]}$, and it is a kind of widespread economic phenomenon in the process of 
economic development. Beijing is located in the upstream of industry chain and has entered the post-industry stage of industrialization, therefore, various resources becoming saturated. In order to further optimize the economic structure, a part of the industry need to transfer out of Beijing. Tianjin is located in the middlestream of industry chain and has entered the advanced stage of industrialization, it is suitable for undertaking aerospace, new energy and new materials, biological medicine and new generation of information technology. While Hebei is located in the downstream of industry chain and is in the intermediate stage of industrialization. It has a solid foundation in the iron and steel, pharmaceutical, textile and other industries with low business costs, cheap labor and basic manufacturing advantages ${ }^{[4]}$. Therefore, through a reasonable industrial links, the Beijing-Tianjin-Hebei region can create a city industrial chain with high competitive advantage, improving the level of manufacturing industry.

Industry transfer has a significant role to the economic growth in the undertaking region, correspondingly, gives rise to a lot of financial needs. Under such circumstances, the industry transfer set a series of higher requirement to the optimization of financial resource allocation, the improvement of the financial organization system and the extension of financial services. Therefore, the study of the financial support of industry transfer among Beijing-Tianjin-Hebei region is very important for solving regional economic integration. This research is beneficial to put forward some countermeasures for risk solving and to realize a stable growth of regional economic, which has important practical significance and theoretical value.

\section{Problems of the Regional Economic Integration of Beijing-Tianjin-Hebei}

The Present Situation of Industry Transfer among Beijing-Tianjin-Hebei. Currently, there are several problems holding back the regional economic integration of Beijing-Tianjin-Hebei. These problems are listed in Table 1.

Table 1 Problems holding back the regional integration of Beijing-Tianjin-Hebei

\begin{tabular}{ll}
\hline Research sites & \multicolumn{1}{c}{ Problems of the regional integration } \\
\hline Beijing city & Large gap of economic level among Beijing-Tianjin-Hebei Region \\
Tianjin city & Lack of overall planning of Beijing-Tianjin-Hebei Region \\
Hebei province & The market function cannot decide the allocation of resources \\
Hebei province & No sound regional cooperation mechanism among the three regions \\
\hline
\end{tabular}

(1) Lack of Overall Planning of Beijing-Tianjin-Hebei Region. From the regional point of view, Beijing, Tianjin and Hebei belong to one region, but actually they belong to three different administrative areas. Therefore, it is difficult to carry out one unified planning for economic development. Due to several reasons such as history, concept and system, three regions are used to do things in their own way, and eager to develop independently. This model of "large and complete" causes the identical industry structure, and the repeated construction results in a serious waste of resources $^{[8]}$.

(2) Large Gap of Economic Level among Beijing-Tianjin-Hebei Region. Being a capital of China, Beijing has stronger attraction on talent and resources than surrounding cities. Due to the above reason, three regions of Beijing-Tianjin -Hebei cannot develop at the same pace, a rare poverty zone around Beijing and Tianjin has occured ${ }^{[9]}$.

(3) The Market Function Cannot Decide the Allocation of Resources. In order to achieve the 
regional economic integration, a complete market economy should be implemented. Under the condition of market-oriented economy, the market can play a decisive role in the allocation of resources. However, the proportion of state-owned economy in Beijing-Tianjin-Hebei region is high, the industrial monopoly is serious and the market awareness is weak, the market segmentation and local protection hinder the free flow of economic resources as well as the transregional economic cooperation $^{[10]}$.

(4) No Sound Regional Cooperation Mechanism among Beijing-Tianjin-Hebei. Firstly, the consultation mechanism for senior leaders among Beijing-Tianjin -Hebei has not been established. In recent years, although the bilateral visits and multilateral negotiations were carried out by top leaders, a formal high-level coordination mechanism have not made significant progress. Secondly, there is no overall cooperation ideas and resultant force among Beijing-Tianjin-Hebei. For a long time, the function of Beijing is to serve the whole nation, this idea makes Beijing cannot focus on regional development.

The Financial Difficulties for the Industry Transfer of Beijing-Tianjin-Hebei. Finance is the core of the economy, so the economic development demands the finance to go ahead first. However, from the present situation of Beijing-Tianjin- Hebei collaboration, there are still many problems need to solve.

(1) The Fuzzy Orientation for Regional Finance Function. Due to the divided regional finance and scattered administrative management, the financial competition is greater than the financial cooperation among Beijing-Tianjin -Hebei region. Both Beijing and Tianjin would like to become the center of regional finance, this kind of situation has become a limiting factor for the financial integration $^{[11]}$.

(2) The Hindering for Financial Integration by Local Interests. The basic requirement for financial integration is the integration of financial market, i.e., the ways, means and environment of financial operation tend to be uniform. Moreover, the orientation and regulation of financial policy must keep pace with each other. All of these require to break the regional segmentation. However, according to the appraisal system for governments at all levels in China, the growth of regional economic is an important indicator. As a result, it is inevitable that the local governments will make decisions from the perspective of local interests, hence, they may intervene in the behavior of credit decision and management of financial institutions directly or indirectly, making the financial integration to be hold back by the local protectionism.

(3) The Economic Imbalance Restricts the Flow of Financial Factors. The resource distribution among Beijing-Tianjin-Hebei region is unbalanced, and the development level is not consistent. The social financing scale of Beijing, Tianjin and Hebei was $64.4 \%, 34.2 \%$ and $22.1 \%$ of GDP (Gross Domestic Product) respectively in 2013. It is obvious that the financial level of Beijing is the highest, Tianjin is higher, but Hebei is the lowest. Due to the distinct gap of the financial total amount and economic support capability among Beijing-Tianjin-Hebei region, the investment return and the cost of capital vary with different region, which reduces the depth and breadth of financial cooperation $^{[12]}$.

(4) Lack of Unified Standard in Financial Industry. Because the financial industry in Beijing, Tianjin and Hebei is managed by different bank departments, the financial business processes, the standard of service and risk, the mechanism of profit allocation are not unified, which leads to a barrier of mutual exchange, mutual recognition and interconnection of financial products. This kind of phenomenon produces two consequences, one is that the long-distance transaction encounters 
difficulties, accordingly, the paying cost increases. Another is that the establishment of credit system becomes lagging behind, and the information sharing among three regions is unable to realize.

\section{Measures}

Broadening the financial channels. In order to avoid the problems of the undertaking for industrial transfer due to the lack of money, the essential thing is to build a multi-level financing platform which enlarges the financial channels. The governments should support small and medium-sized enterprises to be listed on the stock exchange, increasing the proportion of direct financing. The market construction of stock, fund and bond should be accelerated. The invest of private capital should be encouraged, permitting the people of all social strata to invest and share the listed companies so as to solve the fund problem of the enterprises.

Speeding Up the Financial Innovation. According to the demand of enterprise and market during the industry transfer among Beijing-Tianjin-Hebei region, the governments should promote the innovation of financial products and enlarge the service modes. Therefore, an evaluation mechanism for comprehensive customer service and a reasonable mechanism for complaint handling are needed so as to improve the consciousness of financial service.

Strengthening the construction of financial ecological environment. A good credit environment and a perfect credit system are the keys for the establishment of financial ecological environment. The theory of industry economics pointed out earlier that only a good economic environment has been created, the overall development of economic can be promoted. The government of Beijing, Tianjin and Hebei should pay attention to build a reasonable and effective social credit system, and increase the incentive and constraint action of the credit rating system, gradually expand the coverage of personal credit information.

In addition, the connection between the provinces and cities should be strengthened so as to realize the diversified development of financial service system. Thus, the support role of the financial market to the undertaking of industry transfer will become strong.

\section{Acknowledgements}

The research was funded by the key accounting research project from 2015 to 2016 of Financial Bureau of Tianjin and the Accounting Society of Tianjin, China (No. kjkyxm150902).

\section{References}

[1]S. Zhang. Based on cooperative game of Beijing-Tianjin-Hebei regional economic cooperation. Journal of Hebei Normal University (Philosophy and Social Sciences Edition), 34(2011)30-34.

[2]M. Zhang. Beijing-Tianjin-Hebei integration accelerates the transformation of Beijing industry. China economic times, June 19, 2014, version 007.

[3] S. Yan. Integration of the Beijing-Tianjin-Hebei: how to overall planning for enterprises. China business times, March 20, 2014, version 004.

[4] J. Zhang. Investment opportunities under the integration of the Beijing-Tianjin-Hebei region. Foreign Investment in China, 5 (2014) 18-20.

[5]Z. Liu. Beijing-Tianjin-Hebei integration need to break three big barriers. People's BBS, 4 (2014) 
$52-55$.

[6]H. Tao. Accelerated the integration of the Beijing-Tianjin-Hebei region, the game of industry undertaking is carrying out quietly. China trade news, April 1, 2014, version 003.

[7]G. Zhang, S. Wang, S. liu and S. Jia. Research of the coordinated development of Beijing-Tianjin-Hebei based on the industrial docking and transfe. Economy and management, 28 (2014) 14-20.

[8]X. Liang, J. Xie. The evolution, present situation and development of Beijing-Tianjin-Hebei regional economic integration. Journal of Hebei University of Economy and Trade, 30 (2009) 66-69.

[9]J. Sun. Confirming the new position of the coordinated development of Beijing-Tianjin-Hebei. The People's Daily, October 16, 2014, version 020.

[10] Y. Zhang. Discussion on path choice of integration of the Beijing-Tianjin-Hebei region coordinated development. Modern economic management. 36 (2014) 50-53.

[11]Z. Cai. Finance promotes the integration of the Beijing-Tianjin-Hebei region. Financial Economy, 6 (2014) 14-16.

[12]J. Chen. Beijing-Tianjin-Hebei integration and financial cooperation. Chinese finance, 3 (2014) 58-59. 